\title{
Establishment of a Novel Histopathological Classification of High-Grade Serous Ovarian Carcinoma Correlated with Prognostically Distinct Gene Expression Subtypes
}

Ryusuke Murakami, ${ }^{*}$ Noriomi Matsumura, ${ }^{*}$ Masaki Mandai, ${ }^{* \dagger}$ Kosuke Yoshihara, ${ }^{\ddagger}$ Hiroshi Tanabe, ${ }^{\S}$ Hidekatsu Nakai, ${ }^{\dagger}$ Koji Yamanoi, ${ }^{*}$ Kaoru Abiko, ${ }^{*}$ Yumiko Yoshioka, ${ }^{*}$ Junzo Hamanishi, ${ }^{*}$ Ken Yamaguchi, ${ }^{*}$ Tsukasa Baba, ${ }^{*}$ Masafumi Koshiyama, ${ }^{*}$ Takayuki Enomoto, Aikou Okamoto, ${ }^{\S}$ Susan K. Murphy, ${ }^{\llbracket}$ Seiichi Mori,,$"$ Yoshiki Mikami, ${ }^{* *}$ Sachiko Minamiguchi, ${ }^{\dagger \dagger}$ and Ikuo Konishi*

From the Department of Gynecology and Obstetrics, ${ }^{*}$ Kyoto University Graduate School of Medicine, Kyoto, Japan; the Department of Obstetrics and Gynecology, ${ }^{\dagger}$ Kindai University Faculty of Medicine, Osaka, Japan; the Department of Obstetrics and Gynecology, ${ }^{\ddagger}$ Niigata University Graduate School of Medical and Dental Science, Niigata, Japan; the Department of Obstetrics and Gynecology, ${ }^{\S}$ Jikei University School of Medicine, Tokyo, Japan; the Department of Obstetrics and Gynecology, "Duke University, Durham, North Carolina; the Division of Cancer Genomics," Cancer Institute of Japanese Foundation for Cancer Research, Tokyo, Japan; the Department of Diagnostic Pathology, ** Kumamoto University Hospital, Kumamoto, Japan; and the Department of Diagnostic Pathology ${ }^{\dagger \dagger}$ Kyoto University Hospital, Kyoto, Japan

Accepted for publication December 17, 2015.

Address correspondence to Noriomi Matsumura, M.D., Ph.D., Kyoto University Graduate School of Medicine, 54 Shogoin Kawahara-cho, Sakyo-ku, Kyoto, 606-8507 Japan. E-mail: noriomi@kuhp. kyoto-u.ac.jp.

\begin{abstract}
Recently, The Cancer Genome Atlas data revealed four molecular subtypes of high-grade serous ovarian carcinoma (HGSOC) exhibiting distinct prognoses. We developed four novel HGSOC histopathological subtypes by focusing on tumor microenvironment: mesenchymal transition, defined by a remarkable desmoplastic reaction; immune reactive by lymphocytes infiltrating the tumor; solid and proliferative by a solid growth pattern; and papilloglandular by a papillary architecture. Unsupervised hierarchical clustering revealed four clusters correlated with histopathological subtypes in both Kyoto and Niigata HGSOC transcriptome data sets $(P<0.001)$. Gene set enrichment analysis revealed pathways enriched in our histopathological classification significantly overlapped with the four molecular subtypes: mesenchymal, immunoreactive, proliferative, and differentiated $(P<0.0001$, respectively). In $132 \mathrm{HGSOC}$ cases, progression-free survival and overall survival were best in the immune reactive, whereas overall survival was worst in the mesenchymal transition $(P<0.001$, respectively), findings reproduced in 89 validation cases $(P<0.05$, respectively). The CLOVAR_ MES_UP single-sample gene set enrichment analysis scores representing the mesenchymal molecular subtype were higher in paclitaxel responders than nonresponders $(P=0.002)$ in the GSE15622 data set. Taxanecontaining regimens improved survival of cases with high MES_UP scores compared with nontaxane regimens $(P<0.001)$ in the GSE9891 data set. Our novel histopathological classification of HGSOC correlates with distinct prognostic transcriptome subtypes. The mesenchymal transition subtype might be particularly sensitive to taxane. (Am J Pathol 2016, 186: 1103-1113; http://dx.doi.org/10.1016/j.ajpath.2015.12.029)
\end{abstract}

Ovarian carcinoma is the fifth leading cause of death among female malignancies in the United States. ${ }^{1}$ High-grade serous ovarian carcinoma (HGSOC), accounting for $68 \%$ of ovarian carcinoma, is the histological type with the worst prognosis because it is usually diagnosed at an advanced stage. ${ }^{2}$ Chemotherapy with taxane and platinum is typically provided after debulking surgery to eradicate tumor cells, and $75 \%$ of HGSOC cases respond to this initial treatment. However, many patients experience recurrence and eventually succumb to this disease. ${ }^{3}$ Silverberg $^{4}$ proposed a three-tier grading system $(\mathrm{G} 1, \mathrm{G} 2$, and G3) for

\footnotetext{
Supported by KAKENHI (26253080 and 20452336) of The Ministry of Education, Culture, Sports, Science, and Technology grants-in-aid for scientific research of Japan.

This article is being used in as part of Ryusuke Murakami's dissertation at Kyoto University.

Disclosures: None declared.
} 
invasive ovarian carcinoma, including the serous type. This system used architectural patterning, cytologic atypia, and mitotic figures. Low-grade serous ovarian carcinoma, which corresponds to G1, is different from HGSOC, corresponding to G2/G3, both clinically and biologically. Low-grade serous ovarian carcinoma has a good prognosis and carries $K R A S$ and BRAF mutations, whereas HGSOC has a poor prognosis and carries TP53 and BRCA mutations. ${ }^{5}$ Because G2 and G3 are not substantially different clinically and biologically, ${ }^{6,7}$ serous ovarian carcinoma is currently classified into low-grade serous ovarian carcinoma and HGSOC. ${ }^{8}$ Given the diverse prognoses and chemotherapeutic responses, further histopathological classification of HGSOC is warranted for individualization of treatment. ${ }^{9}$ Notably, histopathological classifications proposed so far do not include features of the interaction with stromal cells.

Recently, analyses of gene expression microarray data from The Cancer Genome Atlas (TCGA) project revealed that HGSOC could be classified as one of four gene expression subtypes: mesenchymal, immunoreactive, proliferative, or differentiated. ${ }^{10,11}$ These subtypes were reproducible in an Australian data set $(\mathrm{C} 1, \mathrm{C} 2, \mathrm{C} 5$, and $\mathrm{C} 4$, respectively). ${ }^{12}$ Both studies reported that tumors of mesenchymal phenotype (mesenchymal or $\mathrm{C} 1$ ) had poor prognoses, whereas immunoreactive or $\mathrm{C} 2$ tumors had favorable prognoses. These subtypes have specific histopathological features. The immunoreactive subtype shows prominent infiltration of lymphocytes into the tumor, and the mesenchymal subtype has marked tissue desmoplasia. ${ }^{11,12}$ In this way, HGSOC gene expression subtypes were histopathologically characterized by the interaction between tumor and surrounding cells rather than by the tumor cells themselves.

Our aim was to establish a novel histopathological classification of HGSOC by taking into account the status of the microenvironment, including infiltration of immune cells and stromal features, thereby corresponding to gene expression subtypes relevant to prognosis and biological differences. We show that this novel classification could lead to individualization of chemotherapeutic regimens for HSGOC.

\section{Materials and Methods}

\section{Tissues and Samples}

A total of 221 HGSOC from the ovary, fallopian tube, and peritoneum, without any pretreatment, were analyzed. One hundred cases underwent surgery between 1997 and 2012 at Kyoto University Hospital (Kyoto, Japan), 32 underwent surgery between 1999 and 2009 at Niigata University Hospital (Niigata, Japan), 42 were registered for the JGOG3016 clinical trial ${ }^{13}$ from Jikei University Hospital (Tokyo, Japan), and 47 underwent surgery between 2002 and 2012 from Kindai University Hospital (Osaka, Japan). Sample size $(n=221)$ was determined by information based on a previous report. ${ }^{12}$ Samples were collected after obtaining written informed consent. The study was approved by each of the institutional ethics committees. Hematoxylin and eosin (H\&E)-stained slides from the Kyoto and Niigata HGSOC cases were used to develop a histopathological classification algorithm. The Jikei and Kindai cases served as a validation set for survival analyses. Where available, primary tumors were used for the classification as well as metastatic tumors for cases where only metastatic tumors were available. Tumor-infiltrating lymphocytes were counted with respect to localization inside or outside cancer nests using a representative slide from each case at $\times 400$ magnification. The number of tumorinfiltrating lymphocytes was defined as the median count obtained from five areas showing the most abundant infiltration. Two gynecologic pathologists (R.M. and I.K.) reviewed H\&E slides independently to classify HGSOC. When their histopathological diagnoses were different, the decisions were made by discussion (R.M. and I.K.).

\section{Interobserver Agreement of the Histopathological Diagnosis}

Five of the authors, specializing in gynecologic pathology and oncology, including two board-certified pathologists (Y.M. and S.M.), evaluated microscopic features of cases for histopathological classification. The training set, generated by R.M. and I.K., consists of $20 \mathrm{H} \& \mathrm{E}-$ stained virtual slides from $20 \mathrm{HGSOC}$ cases containing five of each subtype (ie, one representative slide per case). The digital slides were generated using the NanoZoomer Digital Pathology System produced by Hamamatsu Photonics (Hamamatsu, Japan). After reviewing the training set, N.M., T.B., K.Y., Y.M., and S.M. independently reviewed another 28 HGSOC cases and assigned a diagnosis for the histopathological subtype. Then, an interobserver agreement was assessed by six observers (observers A, B, C, D, $\mathrm{E}$, and $\mathrm{F}$ ). The number of evaluated $\mathrm{H} \& \mathrm{E}$ slides had an average of 9.2 (range, 4 to 15 ).

\section{Immunohistochemical Staining for CD8}

Immunohistochemical staining for CD8 was performed as described $^{14}$ and was evaluated similarly to that previously reported. ${ }^{14}$ Briefly, tumor-infiltrating $\mathrm{CD}^{+} \mathrm{T}$ lymphocytes were counted at $\times 400$ magnification and categorized by their localization as intraepithelial or stromal. Five areas with the most abundant infiltration were counted; the median was defined as the number of tumor-infiltrating $\mathrm{CD} 8^{+}$ $\mathrm{T}$ lymphocytes.

\section{Gene Expression Microarray}

Affymetrix data sets GSE9891 HG-U133 plus $2.0^{12}$ and GSE15622 HG-U133A ${ }^{15}$ were from the National Center for Biotechnology Information's Gene Expression Omnibus 
A

MT feature: mesenchymal transition pattern or labyrinthine pattern
with destructive desmoplastic reaction $>10 \%$

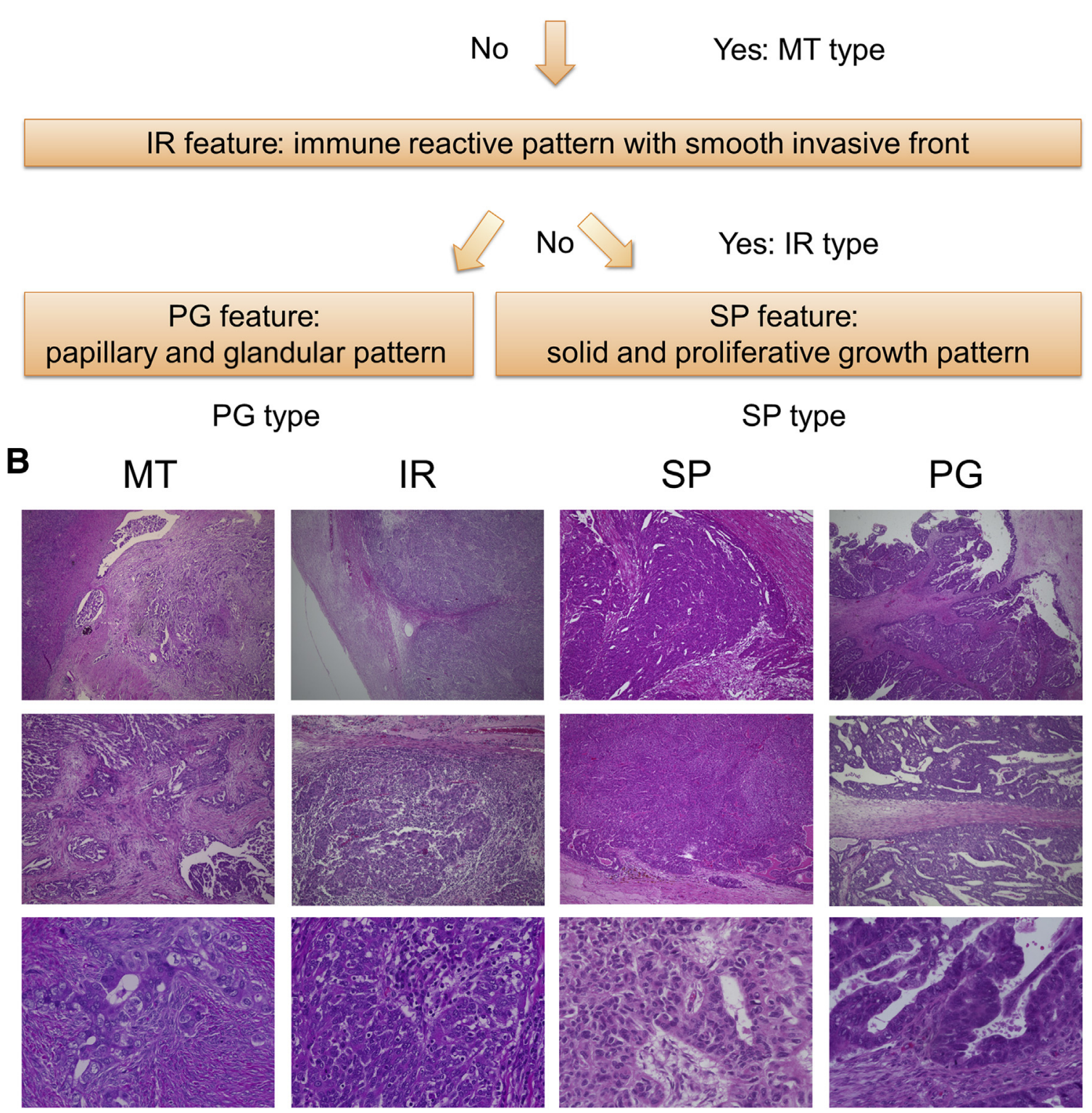

Figure 1 A: The algorithm for the new classification of the four high-grade serous ovarian carcinoma morphological subtypes. B: Morphological findings of the four subtypes. Mesenchymal transition type (MT type) (top panel): Spindle and isolated cells with destructive stromal reaction, referred to as the MT pattern (middle panel); broadly compressed large papillae infiltration with desmoplastic stromal, referred to as the labyrinthine pattern (bottom panel); isolated and invasive cancer cells. Immune reactive type (IR type) (top panel): Relatively round tumor invasive front with tumorinfiltrating lymphocytes (middle panel); a large number of lymphocytes surrounding necrotic tumor (bottom panel); a large number of lymphocytes infiltrating or surrounding tumor. Solid and proliferative type (SP type) (top panel): Proliferative and solid growth architecture (middle panel); proliferative and solid growth architecture with less desmoplastic reaction (bottom panel); solid growth as undifferentiated carcinoma-like. Papilloglandular type (PG type) (top panel): Well-differentiated and papillary growth architecture (middle panel); papillary growth and glandular architecture with less desmoplastic reaction (bottom panel); papillary growth architecture with atypia. Original magnification: $\times 4$ (B, top panels); $\times 10(\mathbf{B}$, middle panels); $\times 40$ (B, bottom panels).

website (http://www.ncbi.nlm.nih.gov/gds, last accessed November 10, 2015). TCGA HG-U133A data set was from the TCGA Data Portal (http://cancergenome.nih.gov, last accessed November 10, 2015). The Affymetrix array data were RMA normalized using the R package affy (http:// www.R-project.org, last accessed November 10, 2015).

We analyzed 27 tumors from 25 Kyoto cases, including two cases with both primary and metastatic tumors, using 


\section{A}

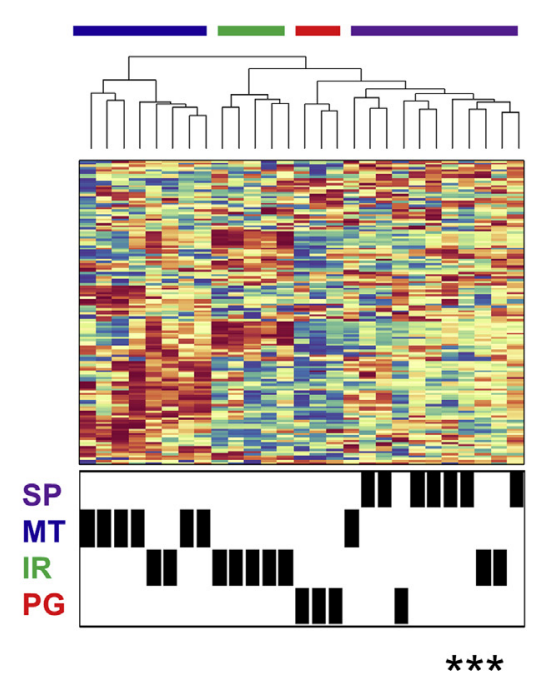

B

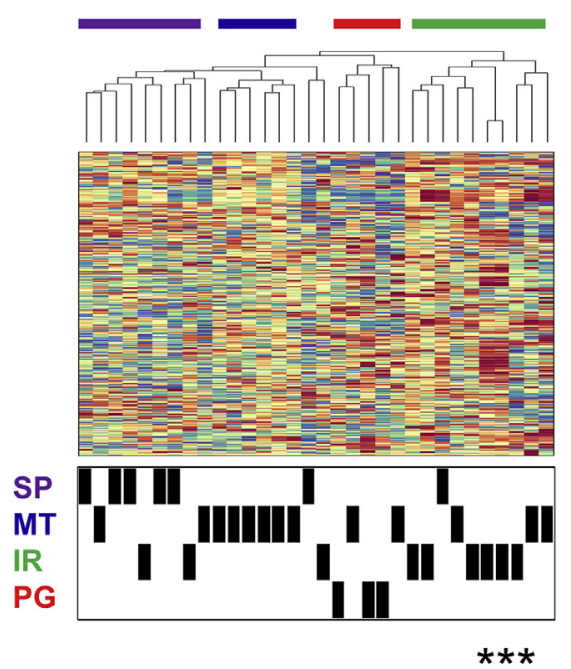

C

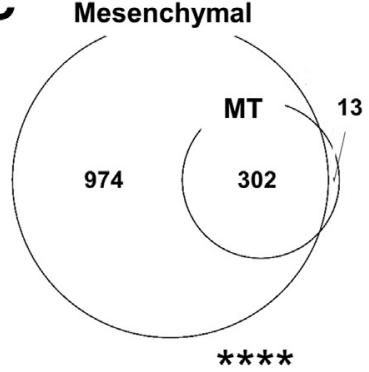

D CLOVAR_MES_UP

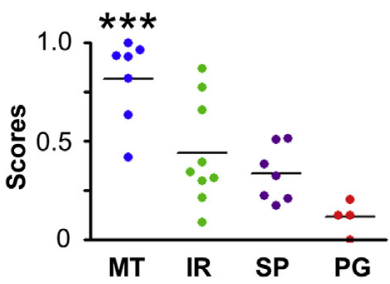

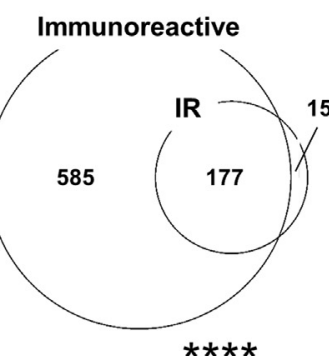

CLOVAR_IMR_UP

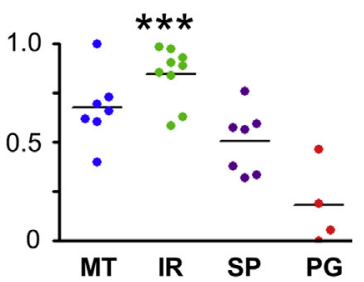

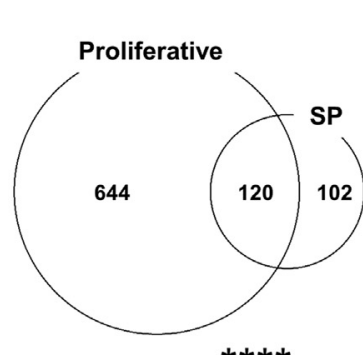

CLOVAR_PRO_UP

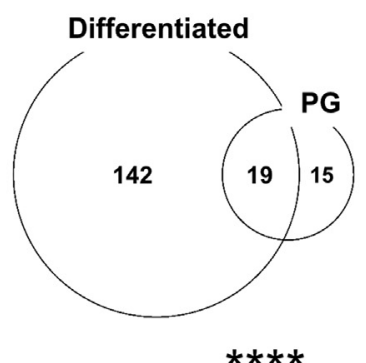

CLOVAR_DIF_UP

Figure 2 Correlation between morphological subtypes and gene expression subtypes. A: Unsupervised hierarchical clustering of the 27 Kyoto high-grade serous ovarian cancer (HGSOC) cases. On the basis of the Affymetrix HG-U133 plus 2.0 microarray data, 117 genes with SD $>1.9$ were used. Morphological subtypes of individual cases are shown as the black bars in the bottom panel. Each of the four tumor clusters highlighted by the horizontal bars in the top panel are significantly enriched with the four morphological subtypes. B: Unsupervised hierarchical clustering of the 32 Niigata HGSOC cases. On the basis of the Agilent Whole Human Genome Microarray $4 \times 44 \mathrm{~K}$ G4112F gene chip microarray data, 489 genes with SD $>1.9$ were used. Similar to the results in A, each of the four tumor clusters were significantly enriched with the four morphological subtypes. C: Number of enriched pathways in the HGSOC subtypes. Gene set enrichment analysis (GSEA) was performed comparing one subtype to the other three subtypes using C2 gene sets version 3.0. We first determined histopathological subtype-specific pathways common to Kyoto and Niigata. Then, the pathways were compared with the pathways enriched in gene expression subtypes in The Cancer Genome Atlas data. The Venn diagrams show significant overlap of the pathways ( $\chi^{2}$ test). D: CLOVAR_MES_UP, IMR_UP, PRO_UP, and DIF_UP single-sample GSEA scores (y axis) were assigned for the 27 Kyoto HGSOC cases. CLOVAR_MES_UP scores were highest in the mesenchymal transition (MT) subtype, whereas CLOVAR_IMR_UP scores were highest in the immune reactive (IR) type. ${ }^{* * *} P<0.001,{ }^{* * * *} P<0.0001 . n=2483$ (C). PG, papilloglandular; SP, solid and proliferative.

the Affymetrix HG-U133 plus 2.0 gene chip, as previously described. ${ }^{16}$ Of the 54,675 gene probes, we selected the 20,853 with annotated gene symbols, and of these, we retained the top half with the highest average expression across the 27 samples. Unsupervised hierarchical clustering with euclidean distance and complete linkage was performed using $\mathrm{R}$ with 136 of the gene probes having an SD >1.9.

We analyzed 32 Niigata cases with the Agilent Whole Human Genome Microarray $4 \times 44 \mathrm{~K}$ G4112F gene chip, as previously described. ${ }^{17}$ The data were normalized with 75 th percentile normalization using GeneSpring GX [11.5]. We used the 29,819 gene probes with annotated gene symbols. For unsupervised hierarchical clustering, 546 gene probes with an SD $>1.9$ were used.

These microarray data are deposited partially in Gene Expression Omnibus (http://www.ncbi.nlm.nih.gov/geo), under accession numbers GSE39204 and GSE55512 for Kyoto and GSE17260 for Niigata microarray data. 
A

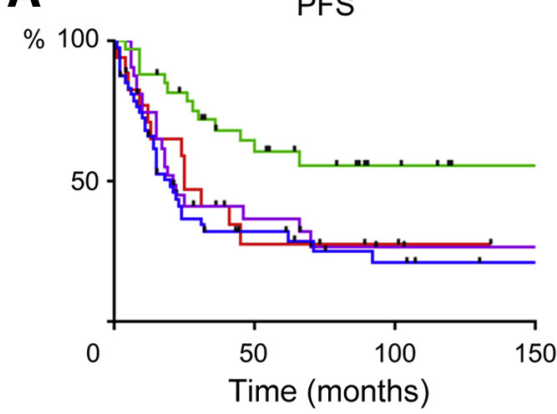

C

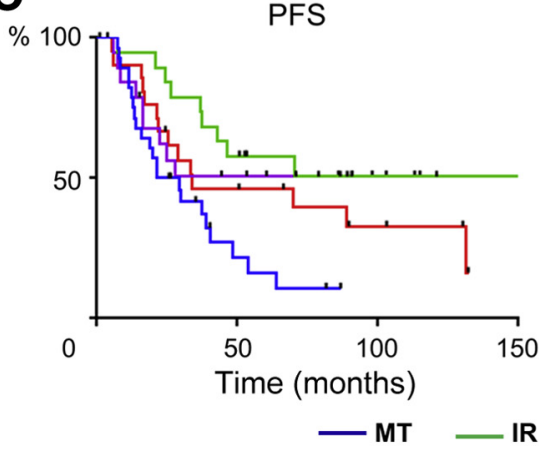

B

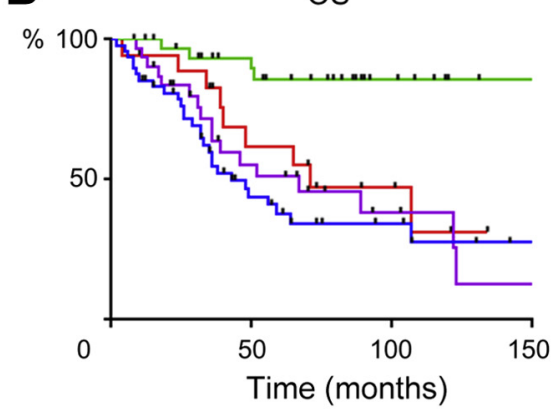

D

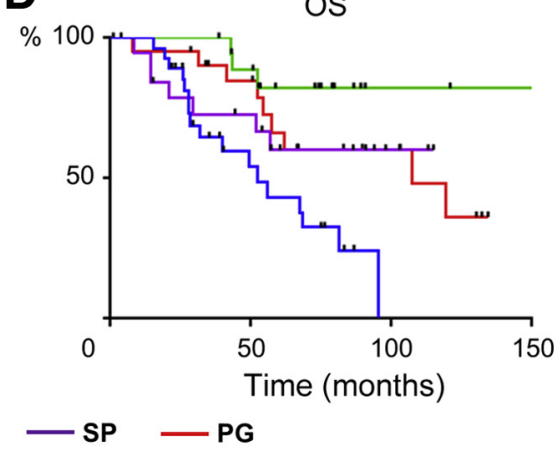

Figure 3 Survival analysis of high-grade serous ovarian cancer cases divided by histopathological subtypes. A and B: Total 132 Kyoto/Niigata cases were classified into mesenchymal transition (MT), immune reactive (IR), solid and proliferative (SP), and papilloglandular (PG). C and D: Total 89 Jikei/Kindai cases were classified into MT, IR, SP, and PG. A: Progression-free survival (PFS) of Kyoto/Niigata cases. IR type PFS was better than the other three types $(P=0.0008)$. B: Overall survival (OS) of Kyoto/Niigata cases. IR type OS was better than the other three types $(P=0.0002)$ and MT type OS was worse than the other three types $(P=0.001)$. C: PFS of Jikei and Kindai cases. IR type PFS was better than the other three types $(P=0.03)$. MT type PFS was worse than the other three types $(P=0.01)$. D: OS of Jikei and Kindai cases. IR type OS was better than the other three types $(P=0.02)$ and MT type OS was worse than the other three types $(P=0.02)$. $n=48$ (A and B, MT type); $n=34$ (A and B, IR type); $n=32$ (A and $\mathbf{B}$, SP type); $n=18$ (A and B, PG type); $n=28$ (C and D, MT type); $n=19$ (C and D, IR type); $n=20$ (C type); $n=22$ (C and D, PG type).

\section{GSEA and SSGSEA}

We downloaded the $\mathrm{C} 2$ curated gene sets version $3.0(n=$ 2483) from the Molecular Signatures Database (http://www. broadinstitute.org/gsea/msigdb, last accessed November 10, 2015) and conducted gene set enrichment analysis (GSEA version 2.1.0; Broad Institute, Cambridge, MA; http://software. broadinstitute.org/gsea) being applied to gene expression microarray data sets with all $\mathrm{C} 2$ gene sets, as described. ${ }^{18}$ The 27 Kyoto cases, the 32 Niigata cases, and the 483 TCGA HGSOC cases were analyzed independently comparing one subtype with the other subtypes. We used the gene expression subtype classifications of the TCGA samples, as previously defined. ${ }^{11}$

Single-sample GSEA (ssGSEA) was performed by gene level, as described (GenePattern version 3.5.0; Broad institute; $h$ ttp://www. broadinstitute.org/cancer/software/ genepattern, last accessed November 10, 2015). ${ }^{19}$ The Classification of Ovarian Cancer (CLOVAR) subtype signature gene sets (CLOVAR_MES_UP, CLOVAR_IMR_UP, CLOVAR_PRO_UP, and CLOVAR_DIF_UP), representative of the gene expression subtypes from the TCGA data set, were used. ${ }^{11}$ For data analyses, the ssGSEA scores were normalized from 0 to 1 .

\section{Statistical Analysis}

GraphPad Prism version 6.0 (GraphPad Software Inc., San Diego, CA) was used for $\chi^{2}$ analysis, unpaired $t$ tests, one-way analysis of variance, and survival analysis. Kaplan-Meier and the Gehan-Breslow-Wilcoxon test were used for survival analysis. $\mathrm{R} \times 64$ version 3.0.2 was used for univariate and multivariate Cox proportional-hazards regression analyses. A
$P<0.05$ was considered significant. For GSEA, false discovery rate $\mathrm{q}<0.25$ was considered significant. Interobserver reproducibility of the histopathological classification was evaluated with Cohen's $\kappa$ coefficient using irr package in R.

\section{Results}

\section{Definition of Four HGSOC Histopathological Subtypes}

We defined an algorithm for HGSOC histopathological classification. TCGA data indicated that genes relevant to immune cells were up-regulated in both the immunoreactive and mesenchymal gene expression subtypes. ${ }^{11}$ Therefore, we selected tumors showing a prominent mesenchymal reaction, followed by selection of tumors with a prominent immune reaction to identify the mesenchymal tumors. A mesenchymal transition (MT) feature was assigned on the basis of the MT pattern or labyrinthine pattern with a remarkable desmoplastic reaction in $>10 \%$ of the tumor area. In more detail, a feature of spindle and isolated cells with destructive stromal reaction is referred to as the MT pattern, and a feature of broadly compressed large papillae infiltration with desmoplastic stroma is referred to as the labyrinthine pattern. If the MT feature was positive, the tumor was defined as being the MT subtype. In tumors other than MT subtype, an immune reactive (IR) feature was assigned when numerous lymphocytes surrounded cancer nests $(>100 / \times 400$ visual field) and infiltrating cancer nests ( $>50 / \times 400$ visual fields) and when this was associated with a smooth invasive front. If the IR feature was positive, the tumor was defined as being the IR subtype. The remaining tumors were classified into the solid and proliferative (SP) 
Table 1 Clinical Characteristics of the 132 HGSOC Cases

\begin{tabular}{|c|c|c|c|c|}
\hline Subtype & MT type & IR type & SP type & PG type \\
\hline No. (\%) & $48(36)$ & $34(26)$ & $32(24)$ & $18(14)$ \\
\hline \multicolumn{5}{|l|}{ Age, years } \\
\hline Mean (minimum-maximum) & $59(39-81)$ & $56(34-77)$ & $62(35-82)$ & $61(43-75)$ \\
\hline$P$ value & 0.96 & 0.04 & 0.18 & 0.43 \\
\hline \multicolumn{5}{|l|}{ Stage } \\
\hline I or II & 4 & 12 & 4 & 4 \\
\hline \multicolumn{5}{|l|}{ Residual disease } \\
\hline Optimal & 27 & 33 & 25 & 14 \\
\hline Suboptimal & 21 & 1 & 7 & 4 \\
\hline$P$ value & 0.0002 & 0.0006 & 0.64 & 0.77 \\
\hline \multicolumn{5}{|l|}{ Chemotherapy } \\
\hline Nontaxane platinum & 2 & 4 & 6 & 3 \\
\hline
\end{tabular}

Statistical analyses were performed comparing one subtype with the other subtypes.

HGSOC, high-grade serous ovarian cancer; IR, immune reactive; MT, mesenchymal transition; NA, not available; PG, papilloglandular; SP, solid and proliferative.

subtype or the papilloglandular (PG) subtype, according to these features (Figure 1).

The number of intraepithelial and stromal lymphocytes seemed to correlate with the number of intraepithelial and stromal $\mathrm{CD} 8^{+}$lymphocytes because the IR subtype had the largest number of $\mathrm{CD}^{+}$cells (Supplemental Figure S1). The number of mitoses did not differ among the subtypes (data not shown).

To determine whether the histopathological subtype changes after metastasis, we defined subtypes using H\&E slides from matched primary and metastatic HGSOC tumors. The subtypes were unchanged between primary and metastatic sites in 49 of $56(88 \%)$ cases (Supplemental Table S1). Notably, the MT subtype did not increase in the metastatic tumors, consistent with a previous report showing the degree of desmoplasia was unchanged in extraovarian sites. ${ }^{12}$ Thus, for HGSOC cases for which the primary sites were not resected at the initial surgery, we defined histopathological subtypes using metastatic tumors.

An interobserver validation study of diagnoses was performed by six observers (Supplemental Table S2). The overall consistency rates of diagnosed MT, IR, SP, and PG had an average of $74 \%$ (range, $61 \%$ to $89 \%$ ). The $\kappa$ coefficients ranged between 0.46 and 0.85 , with an average of 0.64 , and $P$ values were $<0.01$ for all of the pairs. The consistency rates for the diagnoses of MT and the others had an average of $85 \%$ (range, $71 \%$ to $96 \%$ ). The $\kappa$ coefficients ranged between 0.44 and 0.92 , with an average of 0.70 , and $P$ values were $<0.02$ for all of the pairs. The images of problematic cases of MT and other subtypes that produced conflicting assessments can be seen in Supplemental Figures S2, S3, S4, and S5.

\section{Correlation of the Histopathological Subtypes and Gene Expression Profile}

Unsupervised hierarchical clustering using Affymetrix microarray data for the 27 Kyoto HGSOC tumors generated four tumor clusters. These clusters were correlated with the histopathological subtypes $(P<0.001)$ (Figure $2 \mathrm{~A})$. The association between gene expression profiles and histopathological subtype was reproducible in an analysis of Agilent microarray data from 32 Niigata HGSOC tumors $(P<0.001)$ (Figure 2B).

A total of 2483 pathway gene sets were analyzed for enrichment in each subtype using GSEA. Because of the small sample size of our microarray data set, we determined commonly enriched gene sets using both the Kyoto and Niigata data for the histopathological subtype analysis. As a result, 315 gene sets were enriched in MT, 192 in IR, 222 in $\mathrm{SP}$, and 34 in PG. We also conducted GSEA using the TCGA data set and found 1276 gene sets were enriched in the mesenchymal gene expression subtype, 762 in immunoreactive, 764 in proliferative, and 161 in differentiated. There was significant overlap of gene sets between MT and mesenchymal, IR and immunoreactive, SP and proliferative, and PG and differentiated $(P<0.0001$ for each) (Figure 2C). Commonly enriched gene sets among Kyoto, Niigata, and TCGA are listed in Supplemental Tables S3, S4, S5, and S6. In both the MT and the mesenchymal subtype, gene sets related to transforming growth factor (TGF)- $\beta$ and epithelialmesenchymal transition (EMT) were enriched, such as KEGG_TGF_BETA_SIGNALING_PATHWAY, VERRECCHIA_RESPONSE_TO_TGFB1, and ALONSO_ METASTASIS_EMT_UP. In both the IR and the immunoreactive subtypes, gene sets related to the immune 
Table 2 Univariate and Multivariate Cox Proportional Hazards Regression Analysis for Clinical Variables and the Four Morphological Subtypes

\begin{tabular}{|c|c|c|c|c|c|}
\hline Clinical variables & Sample size, no. (\%) & Univariate, HR (95\% CI) & $P$ value & Multivariate, HR (95\% CI) & $P$ value \\
\hline \multicolumn{6}{|l|}{ Age, years } \\
\hline$<60$ & $68(52)$ & 1 & & 1 & \\
\hline$\geqq 60$ & $64(48)$ & $1.4(0.86-2.42)$ & 0.16 & $1.5(0.84-2.49)^{*}$ & 0.18 \\
\hline \multicolumn{6}{|l|}{ Stage } \\
\hline I or II & $24(18)$ & 1 & & 1 & \\
\hline III or IV & $108(82)$ & $3.7(1.33-10.16)$ & 0.01 & $2.8(0.99-7.78)^{*}$ & 0.052 \\
\hline Suboptimal & $33(25)$ & $2.7(1.55-4.53)$ & 0.0004 & $1.8(0.97-3.19)^{*}$ & 0.062 \\
\hline \multicolumn{6}{|l|}{ Chemotherapy } \\
\hline Nontaxane platinum & $15(11)$ & 1 & & 1 & \\
\hline Taxane platinum & $117(89)$ & $0.6(0.33-1.24)$ & 0.19 & $0.5(0.23-0.98)^{*}$ & 0.043 \\
\hline \multicolumn{6}{|l|}{ Subtype } \\
\hline Non SP type & $100(76)$ & 1 & & 1 & \\
\hline SP type & $32(24)$ & $1.5(0.83-2.57)$ & 0.19 & $1.2(0.69-2.23)^{\dagger}$ & 0.47 \\
\hline Non PG type & $114(86)$ & 1 & & 1 & \\
\hline PG type & $18(14)$ & $1(0.50-2.08)$ & 0.82 & $1.1(0.50-2.21)^{\dagger}$ & 0.89 \\
\hline
\end{tabular}

*Multivariate Cox regression analysis of clinical variables with MT type.

${ }^{\dagger}$ For multivariate Cox regression, each subtype was independently analyzed with other clinical variables (age, stage, residual tumor at operation, chemotherapy) from the remaining subtypes.

$H R$, hazard ratio; IR, immune reactive; MT, mesenchymal transition; PG, papilloglandular; SP, solid and proliferative.

response were enriched, including KEGG_T_CELL_ RECEPTOR_SIGNALING_PATHWAY, KEGG_ANTIGEN_ PROCESSING_AND_PRESENTATION, and REACTOME_ SIGNALING_IN_IMMUNE_SYSTEM. In both the SP and the proliferative subtype, gene sets related to proliferation were enriched, such as BENPORATH_PROLIFERATION and KEGG_DNA_REPLICATION. In both the PG and the differentiated subtype, gene sets representing estrogen receptor effects were enriched, such as DOANE_BREAST_ CANCER_ESR1_UP and VANTVEER_BREAST_ CANCER_ESR1_UP.

We next performed CLOVAR ssGSEA scoring using the previously reported gene signatures representative of gene expression subtypes, although the scores did not exclusively point to the subtypes reported in the original article. ${ }^{11}$ CLOVAR_MES_UP scores were high in the MT type $(P=0.001)$, whereas CLOVAR_IMR_UP scores were high in the IR type $(P=0.001)$ in the Kyoto HGSOC tumors (Figure 2D). Regarding the CLOVAR_PRO_UP and CLOVAR_DIF_UP scores, the differences among the four histopathological subtypes were not statistically significant, although the former tended to be high in the SP type and the latter in the PG type (Figure 2D). A similar trend was observed for the MT and IR types in Niigata tumors, but was not significant (Supplemental Figure S6), probably because of the difference of the microarray probes used. ${ }^{11}$ From these analyses, we concluded that the MES_UP and the IMR_UP scores predict their relevant histopathological subtypes, at least from the analysis of the Affymetrix data set.

\section{Correlation of the Histopathological Subtypes with Clinical Data}

We next conducted survival analyses using the HGSOC cases from Kyoto and Niigata $(n=132)$. These were classified into MT $(n=48)$, IR $(n=34), \operatorname{SP}(n=32)$, and PG $(n=18)$ types. Progression-free survival (PFS) for the IR type was much better than the other three types $(P=0.0008)$ (Figure 3A). Similarly, overall survival (OS) for the IR type was markedly better than the other three types $(P=0.0002)$ (Figure 3B). In addition, OS for the MT type was worse than the other three types $(P=0.001)$ (Figure 3B). We next analyzed the Jikei and Kindai validation set $(n=89)$, for which the histopathological classification was performed without any clinical information. These were classified into MT $(n=28)$, IR $(n=19)$, SP $(n=20)$, and PG $(n=22)$ types. Consistent with the analysis of Kyoto/Niigata cases, PFS for the IR type was better than the other three types $(P=0.03)$ (Figure 3C) and PFS for the MT type was worse than the other three types $(P=0.01)$ (Figure 3C). Furthermore, OS for the IR type was better than for the other three types $(P=0.02)$ (Figure $3 \mathrm{D}$ ) and OS for the MT type was worse than the other three $(P=0.02)$ (Figure 3D). Therefore, the association between the histopathological classification and survival was reproducible. 
A

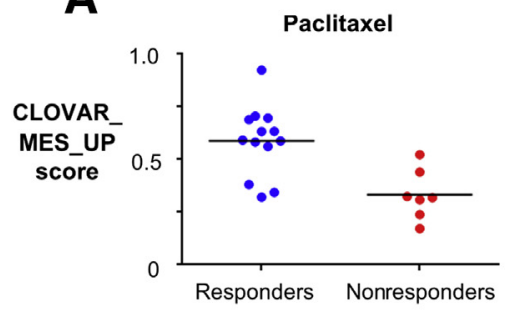

C

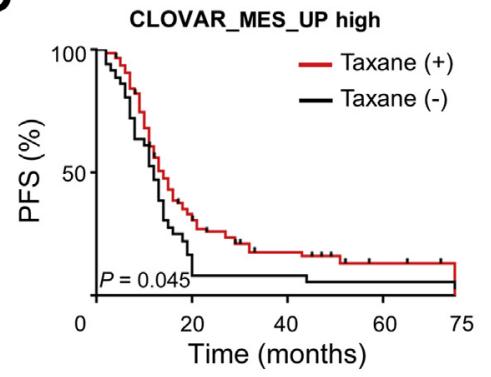

E

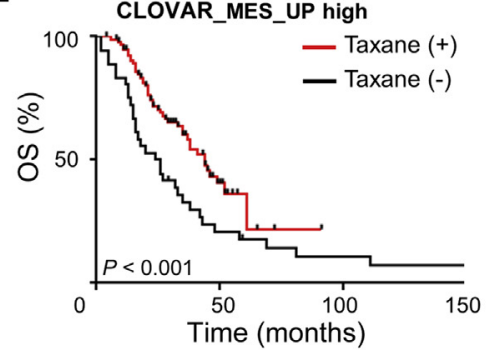

B

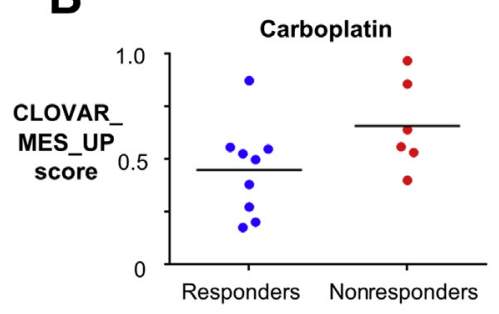

D

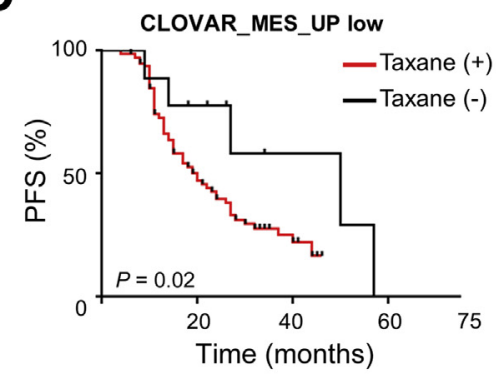

$\mathbf{F}$

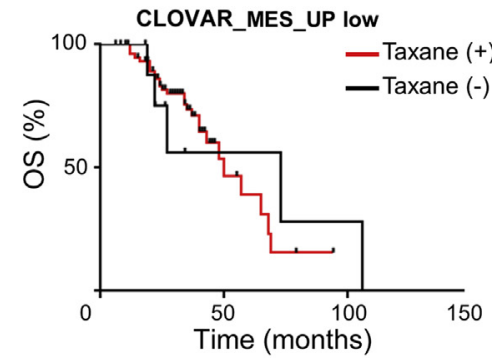

Figure 4 Chemotherapeutic response differences on the basis of CLOVAR_MES_UP single-sample gene set enrichment analysis scores. $\mathbf{A}$ and $\mathbf{B}$ : GSE15622 is an ovarian cancer data set composed of cases for which paclitaxel monotherapy or carboplatin monotherapy was given. Paclitaxel-sensitive cases and resistant cases or carboplatin-sensitive cases and resistant cases were registered. C-F: GSE9891 is an ovarian cancer data set composed of cases that used chemotherapeutic regimens with taxane or without taxane. A: MES_UP scores are higher in the paclitaxel responders $(P=0.002)$. B: MES_UP scores were relatively low in the carboplatin responders $(P=0.09)$. C: In the MES_UP high score $(\geq 0.5)$ group, 96 cases treated with taxane regimens improved progression-free survival (PFS) compared with 36 cases treated with nontaxane regimens $(P=0.045)$. D: In the MES_UP low score $(<0.5)$ group, 80 cases treated with taxane regimens showed a statistically significant decrease in PFS compared with 9 cases treated with nontaxane regimens $(P=0.02)$. E: In the MES_UP high score $(\geq 0.5)$ group, 96 cases treated with taxane regimens improved overall survival (OS) compared with 36 cases treated with nontaxane regimens $(P<0.001)$. F: In the MES_UP low score $(<0.5)$ group, $\mathrm{OS}$ is not significantly different between 80 cases treated with taxane and 9 cases treated with nontaxane regimens. $n=20$ (A and $\mathbf{B}$, paclitaxel monotherapy); $n=15$ (A and $\mathbf{B}$, carboplatin monotherapy); $n=13$ (A and $\mathbf{B}$, paclitaxel sensitive); $n=7$ (A and $\mathbf{B}$, paclitaxel resistant); $n=9$ (A and $\mathbf{B}$, carboplatin sensitive); $n=6$ (A and $\mathbf{B}$, carboplatin resistant); $n=176(\mathbf{C}-\mathbf{F}$, with taxane); $n=45$ (C-F, without taxane).
Next, other clinical variables were analyzed in relation to the histopathological classification using the 132 Kyoto/Niigata cases. We found that the MT type was significantly associated with late stage $(P=0.03)$ and suboptimal debulking $(P<0.001)$. Conversely, the IR type was associated with younger age $(P=0.04)$, early stage $(P=0.003)$, and optimal debulking $(P<0.001)$ (Table 1).

A univariate Cox regression analysis showed that advanced stage (stage III/IV) [hazard ratio (HR), 3.7; 95\% CI, 1.33-10.16; $P=0.01$ ], suboptimal debulking (HR, 2.7; 95\% CI, 1.55-4.53; $P<0.001$ ), and MT type (HR, 2.2; 95\% CI, $1.29-3.62 ; P=0.004)$ were poor prognostic factors. In contrast, IR subtype was a favorable prognostic factor (HR, 0.15; 95\% CI, 0.06-0.43; $P<0.001$ ). In a multivariate Cox regression, the MT subtype was an independent poor prognostic factor (HR, 1.86; 95\% CI, $1.02-3.40 ; P=0.042$ ) and IR subtype was an independent favorable prognostic factor (HR, $0.21 ; 95 \% \mathrm{CI}, 0.07-0.59$; $P=0.003$ ) (Table 2).

\section{Response to Chemotherapy}

We next determined if gene expression subtypes are associated with a particular pattern of response to chemotherapy.
GSE15622 is a gene expression microarray data set composed of laparoscopic biopsy specimens from ovarian cancers that were subsequently treated with paclitaxel monotherapy $(n=20)$ or carboplatin monotherapy $(n=$ 15). ${ }^{15}$ In an ssGSEA analysis, CLOVAR_MES_UP scores were higher in paclitaxel responders than in nonresponders $(P=0.002)$ (Figure $4 \mathrm{~A})$, whereas the scores were lower in carboplatin responders than nonresponders $(P=0.09)$ (Figure 4B). The other gene expression subtype scores did not significantly correlate with response to paclitaxel or carboplatin (Supplemental Figure S7). GSE9891 is a gene expression microarray data set containing HGSOC cases that received chemotherapeutic regimens with taxane $(n=$ 176) or without taxane $(n=45) .{ }^{12}$ In cases with high CLOVAR_MES_UP scores $(\geqq 0.5)$, taxane-containing regimens improved both PFS (Figure $4 \mathrm{C}$ ) and OS (Figure 4E) compared with nontaxane regimens $(P=0.045$ and $P<0.001$, respectively). In contrast, for cases with low CLOVAR_MES_UP scores $(<0.5)$, taxane-containing regimens were associated with decreased PFS compared with nontaxane regimens $(P=0.02)$ (Figure 4D). No differences were observed for OS in the cases with low CLOVAR_MES_UP scores (Figure 4F). These analyses using CLOVAR scores indicate that HGSOC of the mesenchymal gene expression subtype are particularly sensitive to taxane. 


\section{Discussion}

For the first time, we have identified a histopathological classification of HGSOC that correlates with the previously defined gene expression subtypes. ${ }^{11}$ To this end, we developed an algorithm placing emphasis on the interaction between tumor cells and the cellular composition of the surrounding environment. Consistent with prior reports, ${ }^{10-12}$ unsupervised hierarchical clustering of our HGSOC microarray data generated four tumor clusters. These clusters strongly correlated with our four histopathological subtypes (Figure 2, A and B). The pathways common to the histopathological subtypes and their relevant gene expression subtypes matched histopathological features (Supplemental Tables S3, S4, S5, and S6). These results strongly suggest concordance of our histopathological classification and the gene expression subtypes.

Development of a novel histopathological classification scheme that reflects patient prognosis has the potential to enormously affect clinical practice. We found that IR showed good and MT poor OS (Figure 3B and Table 2). Furthermore, these results were reproducible in the Jikei and Kindai validation set (Figure 3D). Our results are also consistent with gene expression subtype analysis reporting good prognosis for immunoreactive and poor prognosis for mesenchymal subtypes. ${ }^{11,12}$ In addition, we and other groups previously reported that an increased T-cell-mediated immune response is related to a favorable prognosis for ovarian cancer patients, by gene expression microarray ${ }^{20}$ and immunohistochemistry of CD8. ${ }^{14,21}$ On the other hand, numerous reports have shown TGF- $\beta$ signaling and EMT is associated with ovarian cancer invasion and metastasis and also, therefore, with poor prognosis. ${ }^{22-24}$

Unlike the OS data, PFS of the MT subtype was similar to SP and PG (Figure 3, A and C), although MT was significantly associated with advanced stage (Table 1 ). This result suggests that the MT type is sensitive to the platinum and taxane chemotherapeutic regimen because $88 \%$ of Kyoto/ Niigata cases and $99 \%$ of Jikei and Kindai cases used this combination. Interestingly, elevated CLOVAR_MES_UP scores were associated with sensitivity to paclitaxel, but relative resistance to carboplatin (Figure 4, A and B). Survival analysis also showed efficacy of taxane in tumors with elevated CLOVAR_MES_UP scores (Figure 4, C-F). Several reports have shown EMT causes platinum resistance in ovarian cancer, ${ }^{25,26}$ pancreatic cancer, ${ }^{27,28}$ and head and neck squamous cell cancer, ${ }^{29}$ whereas EMT was reported to increase sensitivity to paclitaxel by down-regulating $\beta \mathrm{III}$ and $\beta I V$ a tubulin in lung cancer. ${ }^{30}$ Our data suggest that taxane is more effective against tumors with a MT phenotype in ovarian cancer, although the precise mechanisms remain to be elucidated.

Although the platinum and taxane combination is an effective regimen against ovarian cancer, few reports address individualization of chemotherapeutics by predicting sensitivity to each drug. Our data suggest that the effective agent in the paclitaxel and carboplatin regimen may be different from patient to patient, and gene expression microarray analysis of HGSOC would enable individualization of chemotherapeutics. Even if gene expression microarray data were not available, our novel histopathological classification of HGSOC could, on its own, enable such individualization. That is, a taxane-based regimen would be effective against the mesenchymal or MT type.

There are several limitations in this study. First, the reproducibility of the diagnoses of the four HGSOC subtypes was statistically significant, but was considered to be suboptimal for practical use (Supplemental Table $\mathrm{S} 2$ ). We described in detail the causes of disagreement (Supplemental Figures S2, S3, S4, and S5), and summarized points to improve reproducibility. It should be emphasized that the distinction between MT type and non-MT type is vital, because identification of the former prompts the individualization of treatment, as described above. Therefore, the two-tier classification might be practical. A refinement of the criteria established for the current study appears to be essential for better interobserver agreements for clinical use. Second, high-grade serous carcinomas can be morphologically heterogeneous, and thus it appears difficult to determine the optimal number of $\mathrm{H} \& \mathrm{E}$ slides for classification, although we reviewed all slides, mostly nine or more per case for classification. Further studies should determine the number of slides for better interobserver agreements. Third, the number of cases with both H\&E slides and microarray data available was limited. Although the pathway analysis produced reasonable results (Figure 2C), a comparison between morphological subtypes and scoring from molecular subtype signatures (Figure 2D) should be performed in a larger cohort in the future. The limited number may have caused statistically insignificant results of CLOVAR_PRO_UP scores and CLOVAR_DIF_UP scores.

In conclusion, we generated a novel histopathological classification of HGSOC that correlates with gene expression subtypes and prognosis, and thus, that can be recommended for clinical use. In addition, we found that the poor prognostic mesenchymal gene expression subtype, corresponding to the MT type, is sensitive to taxane. This study serves as a milestone in the development of individualized medicine against ovarian cancer.

\section{Acknowledgments}

We thank Yuko Hosoe (Kyoto University) for her excellent technical assistance; Ryo Yamada, Maiko Narahara, Koichiro Higasa, Chikashi Terao, Fumihiko Matsuda, Zhipeng Wang (Kyoto University), Tetsuya Sato (Kyushu 
University), and Tan Tuan Zea (National University of Singapore) for their excellent bioinformatics advice and assistance; and Kenichi Tanaka, Yuki Hata (Niigata University), Motoaki Saito, Akihiko Misawa, and Masataka Takenaka (Jikei University School of Medicine, Tokyo, Japan) for the hematoxylin and eosin slides used in this study.

\section{Supplemental Data}

Supplemental material for this article can be found at http://dx.doi.org/10.1016/j.ajpath.2015.12.029.

\section{References}

1. Siegel RL, Miller KD, Jemal A: Cancer statistics, 2015. CA Cancer J Clin 2015, 65:5-29

2. Seidman JD, Horkayne-Szakaly I, Haiba M, Boice CR, Kurman RJ, Ronnett BM: The histologic type and stage distribution of ovarian carcinomas of surface epithelial origin. Int J Gynecol Pathol 2004, 23 : $41-44$

3. Miller DS, Blessing JA, Krasner CN, Mannel RS, Hanjani P, Pearl ML, Waggoner SE, Boardman CH: Phase II evaluation of pemetrexed in the treatment of recurrent or persistent platinumresistant ovarian or primary peritoneal carcinoma: a study of the Gynecologic Oncology Group. J Clin Oncol 2009, 27: 2686-2691

4. Silverberg SG: Histopathologic grading of ovarian carcinoma: a review and proposal. Int J Gynecol Pathol 2000, 19:7-15

5. Vang R, Shih Ie M, Kurman RJ: Ovarian low-grade and high-grade serous carcinoma: pathogenesis, clinicopathologic and molecular biologic features, and diagnostic problems. Adv Anat Pathol 2009, 16: 267-282

6. Malpica A, Deavers MT, Lu K, Bodurka DC, Atkinson EN, Gershenson DM, Silva EG: Grading ovarian serous carcinoma using a two-tier system. Am J Surg Pathol 2004, 28:496-504

7. Singer G, Kurman RJ, Chang HW, Cho SK, Shih Ie M: Diverse tumorigenic pathways in ovarian serous carcinoma. Am J Pathol 2002, 160:1223-1228

8. Bodurka DC, Deavers MT, Tian C, Sun CC, Malpica A, Coleman RL, Lu KH, Sood AK, Birrer MJ, Ozols R, Baergen R, Emerson RE, Steinhoff M, Behmaram B, Rasty G, Gershenson DM: Reclassification of serous ovarian carcinoma by a 2-tier system: a Gynecologic Oncology Group Study. Cancer 2012, 118:3087-3094

9. Bowtell DD: The genesis and evolution of high-grade serous ovarian cancer. Nat Rev Cancer 2010, 10:803-808

10. Cancer Genome Atlas Research Network: Integrated genomic analyses of ovarian carcinoma. Nature 2011, 474:609-615

11. Verhaak RG, Tamayo P, Yang JY, Hubbard D, Zhang $H$, Creighton CJ, et al; Cancer Genome Atlas Research Network: Prognostically relevant gene signatures of high-grade serous ovarian carcinoma. J Clin Invest 2013, 123:517-525

12. Tothill RW, Tinker AV, George J, Brown R, Fox SB, Lade S, Johnson DS, Trivett MK, Etemadmoghadam D, Locandro B, Traficante N, Fereday S, Hung JA, Chiew YE, Haviv I; Australian Ovarian Cancer Study Group, Gertig D, DeFazio A, Bowtell DD: Novel molecular subtypes of serous and endometrioid ovarian cancer linked to clinical outcome. Clin Cancer Res 2008, 14: $5198-5208$

13. Katsumata N, Yasuda M, Takahashi F, Isonishi S, Jobo T, Aoki D, Tsuda H, Sugiyama T, Kodama S, Kimura E, Ochiai K, Noda K; Japanese Gynecologic Oncology Group: Dose-dense paclitaxel once a week in combination with carboplatin every 3 weeks for advanced ovarian cancer: a phase 3, open-label, randomised controlled trial. Lancet 2009, 374:1331-1338

14. Hamanishi J, Mandai M, Iwasaki M, Okazaki T, Tanaka Y, Yamaguchi K, Higuchi T, Yagi H, Takakura K, Minato N, Honjo T, Fujii S: Programmed cell death 1 ligand 1 and tumor-infiltrating CD8 ${ }^{+}$ T lymphocytes are prognostic factors of human ovarian cancer. Proc Natl Acad Sci U S A 2007, 104:3360-3365

15. Ahmed AA, Mills AD, Ibrahim AE, Temple J, Blenkiron C, Vias M, Massie CE, Iyer NG, McGeoch A, Crawford R, Nicke B, Downward J, Swanton C, Bell SD, Earl HM, Laskey RA, Caldas C, Brenton JD: The extracellular matrix protein TGFBI induces microtubule stabilization and sensitizes ovarian cancers to paclitaxel. Cancer Cell 2007, 12: $514-527$

16. Abiko K, Mandai M, Hamanishi J, Yoshioka Y, Matsumura N, Baba T, Yamaguchi K, Murakami R, Yamamoto A, Kharma B, Kosaka K, Konishi I: PD-L1 on tumor cells is induced in ascites and promotes peritoneal dissemination of ovarian cancer through CTL dysfunction. Clin Cancer Res 2013, 19:1363-1374

17. Yoshihara K, Tajima A, Yahata T, Kodama S, Fujiwara H, Suzuki M, Onishi Y, Hatae M, Sueyoshi K, Fujiwara H, Kudo Y, Kotera K, Masuzaki H, Tashiro H, Katabuchi H, Inoue I, Tanaka K: Gene expression profile for predicting survival in advanced-stage serous ovarian cancer across two independent datasets. PLoS One 2010, 5: e9615

18. Subramanian A, Tamayo P, Mootha VK, Mukherjee S, Ebert BL, Gillette MA, Paulovich A, Pomeroy SL, Golub TR, Lander ES, Mesirov JP: Gene set enrichment analysis: a knowledge-based approach for interpreting genome-wide expression profiles. Proc Natl Acad Sci U S A 2005, 102:15545-15550

19. Barbie DA, Tamayo P, Boehm JS, Kim SY, Moody SE, Dunn IF, Schinzel AC, Sandy P, Meylan E, Scholl C, Frohling S, Chan EM, Sos ML, Michel K, Mermel C, Silver SJ, Weir BA, Reiling JH, Sheng Q, Gupta PB, Wadlow RC, Le H, Hoersch S, Wittner BS, Ramaswamy S, Livingston DM, Sabatini DM, Meyerson M, Thomas RK, Lander ES, Mesirov JP, Root DE, Gilliland DG, Jacks T, Hahn WC: Systematic RNA interference reveals that oncogenic KRAS-driven cancers require TBK1. Nature 2009, 462: $108-112$

20. Yoshihara K, Tsunoda T, Shigemizu D, Fujiwara H, Hatae M, Fujiwara H, Masuzaki H, Katabuchi H, Kawakami Y, Okamoto A, Nogawa T, Matsumura N, Udagawa Y, Saito T, Itamochi H, Takano M, Miyagi E, Sudo T, Ushijima K, Iwase H, Seki H, Terao Y, Enomoto T, Mikami M, Akazawa K, Tsuda H, Moriya T, Tajima A, Inoue I, Tanaka K; Japanese Serous Ovarian Cancer Study Group: High-risk ovarian cancer based on 126-gene expression signature is uniquely characterized by downregulation of antigen presentation pathway. Clin Cancer Res 2012, 18: $1374-1385$

21. Hwang WT, Adams SF, Tahirovic E, Hagemann IS, Coukos G: Prognostic significance of tumor-infiltrating T cells in ovarian cancer: a meta-analysis. Gynecol Oncol 2012, 124:192-198

22. Yang D, Sun Y, Hu L, Zheng H, Ji P, Pecot CV, Zhao Y, Reynolds S, Cheng H, Rupaimoole R, Cogdell D, Nykter M, Broaddus R, Rodriguez-Aguayo C, Lopez-Berestein G, Liu J, Shmulevich I, Sood AK, Chen K, Zhang W: Integrated analyses identify a master microRNA regulatory network for the mesenchymal subtype in serous ovarian cancer. Cancer Cell 2013, 23:186-199

23. Cao L, Shao M, Schilder J, Guise T, Mohammad KS, Matei D: Tissue transglutaminase links TGF-beta, epithelial to mesenchymal transition and a stem cell phenotype in ovarian cancer. Oncogene 2012, 31: 2521-2534

24. Cheon DJ, Tong Y, Sim MS, Dering J, Berel D, Cui X, Lester J, Beach JA, Tighiouart M, Walts AE, Karlan BY, Orsulic S: A collagenremodeling gene signature regulated by TGF-beta signaling is associated with metastasis and poor survival in serous ovarian cancer. Clin Cancer Res 2014, 20:711-723 
25. Yew KH, Crow J, Hirst J, Pressetto Z, Godwin AK: Epimorphininduced MET sensitizes ovarian cancer cells to platinum. PLoS One 2013, 8:e72637

26. Haslehurst AM, Koti M, Dharsee M, Nuin P, Evans K, Geraci J, Childs T, Chen J, Li J, Weberpals J, Davey S, Squire J, Park PC, Feilotter H: EMT transcription factors snail and slug directly contribute to cisplatin resistance in ovarian cancer. BMC Cancer 2012, 12:91

27. Tan J, You Y, Xu T, Yu P, Wu D, Deng H, Zhang Y, Bie P: Par-4 downregulation confers cisplatin resistance in pancreatic cancer cells via PI3K/Akt pathway-dependent EMT. Toxicol Lett 2014, 224:7-15

28. Arumugam T, Ramachandran V, Fournier KF, Wang H, Marquis L, Abbruzzese JL, Gallick GE, Logsdon CD, McConkey DJ, Choi W:
Epithelial to mesenchymal transition contributes to drug resistance in pancreatic cancer. Cancer Res 2009, 69:5820-5828

29. Hsu DS, Lan HY, Huang CH, Tai SK, Chang SY, Tsai TL, Chang CC, Tzeng CH, Wu KJ, Kao JY, Yang MH: Regulation of excision repair cross-complementation group 1 by Snail contributes to cisplatin resistance in head and neck cancer. Clin Cancer Res 2010, 16:4561-4571

30. Tamura D, Arao T, Nagai T, Kaneda H, Aomatsu K, Fujita Y, Matsumoto K, De Velasco MA, Kato H, Hayashi H, Yoshida S, Kimura H, Maniwa Y, Nishio W, Sakai Y, Ohbayashi C, Kotani Y, Nishimura Y, Nishio K: Slug increases sensitivity to tubulin-binding agents via the downregulation of $\beta$ III and $\beta$ IVa-tubulin in lung cancer cells. Cancer Med 2013, 2:144-154 